\title{
Pneumonia Associada à Ventilação Mecânica: Análise de Fatores Epidemiológicos na Confecção de Estratégias de Profilaxia e Terapêutica*
}

\author{
Ventilator Associated Pneumonia: Confection of a Strategy of Prophylaxis \\ and Management Based on the Analysis of Epidemiology
}

Edgard do Carmo Netoํㅜ, Paulo César de Souza², Frederico Azevedo³, Marcelo Elisio Lugarinho4.

\section{RESUMO}

JUSTIFICATIVA E OBJETIVOS: Tem-se creditado à pneumonia associada à ventilação mecânica (PAVM) impacto variável, em termos de mortalidade, aumento da permanência hospitalar e tempo de ventilação mecânica. O objetivo deste estudo foi definir três aspectos: mortalidade e incidência de PAVM antes e após a implantação de protocolo para profilaxia de pneumonia (primários); mapeamento microbiológico (secundário) como instrumento de otimização terapêutica.

MÉTODO: Foi realizada uma coorte histórica de agosto de 2001 a janeiro de 2004, dividida em segmentos pré (até janeiro de $2003, n=52$ ) e pós-implantação do protocolo, com análise de mortalidade e mapeamento microbiológico executados no segundo segmento (subgrupo controle $\mathrm{n}=39$ e subgrupo caso $n=14$ ).

1. Pós-Graduando AMIB do CTI Geral do Hospital das Clínicas Mário Lioni, Médico Rotina do CTI Geral do Hospital São Lucas e Oficial Médico Socorrista do $1^{\circ}$ Grupamento de Socorro de Emergência do Corpo de Bombeiros Militar-RJ.

2. Coordenador dos CTI Gerais do Hospital das Clínicas de Niterói e do Hospital das Clínicas Mário Lioni.

3. Pós-Graduado AMIB no CTI Geral do Hospital de Clínicas Mário Lioni, Médico Rotína do CTI do Hospital Copa D’Or.

4. Médico Rotína do CTI Geral do Hospital de Clínicas Mário Lioni.

*Recebido do Hospital de Clínicas Mário Lioni, Duque de Caxias, RJ.

Apresentado em 05 de fevereiro de 2006

Aceito para publicação em 13 de novembro de 2006

Endereço para correspondência:

Dr. Edgard do Carmo Neto

Travessa Frederico Pamplona, 32

Centro de Terapia Intensiva - Copacabana

22061-080, Rio de Janeiro, RJ.

Fone: (021) 2545-4153

E-mail: rio14312@terra.com.br

(C)Associação de Medicina Intensiva Brasileira, 2006
RESULTADOS: As taxas de incidência nos anos de 2001 a 2003 foram, respectivamente 28,05\% $\pm 12,92 \%$, $22,45 \%$ o $10,18 \%$ o e $10,75 \%$ o $\pm 7,61 \%$. A redução de incidência após a intervenção não alcançou significância estatística $(p>0,4)$. As taxas de mortalidade foram $49 \%$ no grupo controle $\left(\mathrm{IC}_{95}=33 \%\right.$ a $65 \%$ ) e $43 \%$ no grupo caso (IC ${ }_{95}=14 \%$ a $72 \%$ ), com $R R=0,88\left(I C_{95}=0,26\right.$ a 2,94), sem significância estatística $(p=0,65)$. Oito lavados bronco-alveolares foram obtidos (57\%), 50\% com flora mista. Pseudomonas aeruginosa foi isolada em seis pacientes (75\%), Acinetobacter sp em um caso (12,5\%) e Staphylococcus aureus meticilina-resistente (MRSA) em um caso (12,5\%). Outros Gram-negativos produtores de betalactamase de espectro estendido (ESBL) em dois casos (25\%) e Stenotrophomonas maltophilia em um caso $(12,5 \%)$.

CONCLUSÕES: A incidência de PAVM mostrou tendência à redução considerável após a implantação do protocolo de profilaxia, porém os resultados sugerem não haver impacto na mortalidade, sendo necessário estudo prospectivo de maior amostra para conclusões definitivas. Os germes isolados foram, em sua maioria, de alto risco para resistência a antimicrobianos. Assim, é necessário conhecer o perfil de sensibilidade da unidade para traçar estratégia terapêutica inicial adequada.

Unitermos: controle de infecção, epidemiologia, infecção cruzada, pneumonia, respiração artificial.

\section{SUMMARY}

BACKGROUND AND OBJECTIVES: Variable magnitude of impact on the outcomes of the critically ill patients has been credited to ventilation-associated pneumonia, in terms of mortality, length of hospital stay and mechanic ventilation days. Three objecti- 
ves have been defined in this study: mortality and incidence of ventilation-associated pneumonia before and after the implantation of a prophylaxis protocol (primary objectives); microbiologic mapping (secondary objective) as an instrument to optimize therapy.

METHODS: A historical cohort was followed during the period of August 2001 to January 2004, fragmented in two segments, pre (until January 2003, $n=52$ ) and post-implantation of the mentioned protocol, the analysis of mortality and microbiologic mapping been performed in the second segment (control group $n=$ 39 and case group $n=14$ ).

RESULTS: The incidence rates from 2001 to 2003 were respectively $28.05 \%$ o $12.92 \%$, $22.45 \%$ o \pm $10.18 \%$ ond $10.75 \%$ o $\pm 7.61 \%$. The decrease in this rate after the intervention did not reach statistical significance $(p>0.4)$. Mortality rates were $49 \%$ in the control group ( $\mathrm{Cl}_{95}=33 \%$ to $65 \%$ ) and $43 \%$ in the case group $\left(\mathrm{Cl}_{95}=14 \%\right.$ to $\left.72 \%\right), \mathrm{OR}=0.88\left(\mathrm{Cl}_{95}=\right.$ 0.26 to 2.94), without statistical significance either ( $p$ $=0.65$ ). Eight bronco-alveolar lavage were obtained (57\%), 50\% with multiple flora. Pseudomonas aeruginosa was isolated in six patients $(75 \%)$, Acinetobacter $\mathrm{sp}$ in one case $(12.5 \%)$ and methicilin-resistent Staphylococcus aureus (MRSA) in one (12.5\%). Other Gram negative bacilli producers of extended spectrum betalactamase (ESBL) were isolated in two cases (25\%) and Stenotrophomonas maltophilia in another (12.5\%).

CONCLUSIONS: The incidence rate of ventilation-associated pneumonia revealed a tendency to considerable reduction after the utilization of the prophylaxis protocol, while the results suggest no impact on mortality rates. Further prospective evaluation of a greater sample is required, in order to get to definite conclusions regarding prognosis. The isolated germs were, in its majority, high risk pathogens to multiresistance to antibiotics, pointing the necessity of knowledge of local susceptibility profile, so that an adequate initial therapeutic strategy can be undertaken.

Key Words: cross infection, epidemiology, infection control, pneumonia, artificial respiration.

\section{INTRODUÇÃO}

Desde o início da terapia intensiva uma das mais importantes complicações das que se listam durante internação em unidades desta modalidade terapêutica é a pneumonia hospitalar. Destas, a maioria é as- sociada a dispositivo invasivo, isto é, relacionada à ventilação mecânica, como demonstrado mais uma vez recentemente pelo National Nosocomial Infection Survaillance System (NNIS), compondo $85 \%$ das pneumonias nosocomiais ${ }^{1}$.

No início da ventilação mecânica as taxas de pneumonia eram muito elevadas, tendo àquela época como principal fator de risco a contaminação dos fluidos dos reservatórios dos nebulizadores ${ }^{2}$. Ainda hoje, porém, a pneumonia hospitalar, principalmente associada à ventilação mecânica (PAVM), representa a primeira a segunda área mais comum de infecção hospitalar (variando entre $27 \%$ e $47 \%$ delas, sendo a primeira proveniente do último relatório do NNIS do CDC Center for Disease Control and Prevention $^{1}$ e a última do estudo europeu EPIC ${ }^{3}$, passando para $28,9 \%$ em estudo multicêntrico brasileiro) ${ }^{4}$.

Vários estudos de prognóstico têm demonstrado resultados contraditórios do impacto imposto por esta infecção hospitalar, em termos de mortalidade absoluta, relativa e atribuível, aumento dos tempos de permanência hospitalar e de ventilação mecânica ${ }^{5-8}$.

O objetivo deste estudo foi proceder à análise de prognóstico da PAVM, tendo por metas primárias a mortalidade absoluta e relativa. Assim como a aferição da eficácia de um protocolo de profilaxia através das taxas de incidência desta infecção. O objetivo secundário foi o mapeamento da flora responsável, utilizando-o como instrumento de otimização da terapêutica.

\section{MÉTODO}

Foi avaliada uma coorte histórica de pacientes submetidos à ventilação mecânica por período não inferior a 48 horas (critério de exclusão), em unidade intensiva privada de 12 leitos, dos quais a distribuição de ocupação revela $60 \%$ de diagnósticos clínicos à internação, com taxa de densidade de utilização de ventilação mecânica em torno de $30 \%$. A coorte teve início em agosto de 2001 e se estendeu até janeiro de 2004, sendo fragmentada em dois segmentos cronológicos, antes e depois da implantação do protocolo de profilaxia de pneumonia associada à ventilação mecânica, que teve início em janeiro de 2003. O segundo segmento (compreendido entre janeiro de 2003 e janeiro de 2004), no qual fez-se a análise da mortalidade e mapeamento microbiológico, foi ainda dividido em dois grupos: grupo controle, 
composto pelos pacientes que não desenvolveram pneumonia, e grupo caso, integrado por aqueles que a desenvolveram.

O protocolo de prevenção foi idealizado de modo a ser de simples execução, visando à elevada adesão dos profissionais que prestam assistência ventilatória na unidade. Fundamenta-se no tripé: posição semirecumbente no leito (ângulo formado pela linha médio axilar, até o grande trocânter do fêmur e o plano horizontal do leito, a mais de 45 graus, medido por goniômetro impresso de fábrica na junta de articulação de cabeceira de todos os 12 leitos elétricos da unidade $)^{9-12}$, aspiração com técnica asséptica e intubação endotraqueal com técnica e paramentação padronizados com intuito de reduzir qualquer contato do tubo traqueal com quaisquer superfícies contaminadas que não a própria cavidade orofaríngea do paciente. $O$ treinamento na aferição e execução da posição semi-recumbente foi realizado ao término de trabalho de auditoria postural anteriormente realizado nesta unidade ${ }^{9}$.

Os critérios para definição de pneumonia associada à ventilação adotados foram os do $C D C{ }^{13}$, as discordâncias foram dirimidas por dois revisores, médicos integrantes da rotina do serviço. Os diagnósticos foram ainda divididos em casos de início precoce (menos de cinco dias de intubação) e casos de início tardio (após o quinto dia de intubação), tendo sua flora, quando isolada, devidamente registrada.

Durante a avaliação diagnóstica dos casos suspeitos de PAVM o diagnóstico microbiológico é encorajado, porém não obrigatório, através da coleta de lavado bronco-alveolar e cultura quantitativa, considerando positividade como maior que $10^{4} \mathrm{UFC} / \mathrm{mL}^{5-7,14}$.

As variáveis categóricas foram relatadas como proporção e intervalo de confiança de $95 \%$ (IC ${ }_{95}$ ) e as variáveis quantitativas descritas através da média, mediana, desvio-padrão (DP) e IC ${ }_{95}$.

As características de base idade e gravidade de doença à admissão, aferida pelo escore APACHE II, foram comparadas e utilizadas em ajustamento univariável entre os dois grupos do segundo segmento da coorte.

Os testes de hipótese realizados para estimar a significância de diferenças entre as variáveis de base e de desfecho foram: Qui-quadrado $\left(\chi_{2}\right)$ para variáveis categóricas dicotômicas, razão $\mathrm{F}$ e teste $t$ de Student para variáveis quantitativas contínuas. O nível significativo foi arbitrado em 0,05.

\section{RESULTADOS}

Foram seguidos, no total, 105 pacientes: 52 no período de 2001 a 2002, incluindo 25 casos de PAVM e 53 durante o segundo segmento cronológico da coorte (período 2003 a 2004), 14 dos quais desenvolveram PAVM (grupo caso).

Quanto ao segundo segmento temporal da coorte, o tempo médio de ventilação foi de 23 dias (mediana = $12, \mathrm{DP}=29$ e IC ${ }_{95}=15$ a 31 dias) e de permanência na UTI 29 dias (mediana $=20, \mathrm{DP}=29$ e IC $95=21$ a 37 dias). As médias das variáveis de base idade e APACHE II neste segmento foram 61 anos (DP $=20$

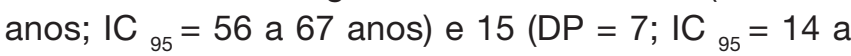
17), respectivamente. $O$ teste $T$ para amostras homocedásticas revelou que entre estas variáveis de base não foram encontradas diferenças estatísticas significativas ( $p=0,43$ e $p=0,71$, respectivamente), entre os grupos caso e controle (Tabela 1). Atente-se ainda para a distribuição de freqüência das categorias de pacientes, isto é, clínicos, cirúrgicos e vítimas de trauma (Figuras 1 e 2). Houve preponderância dos diagnósticos primários: transtornos respiratórios, neurológicos e trauma (Tabela 2). Embora tenha havido predomínio de pacientes clínicos na amostra, dentre os pacientes que desenvolveram PAVM há uma proporção maior de vítimas de trauma (17\% versus $29 \%$, respectivamente).

Tabela 1 - Características de Base Registradas nos Dois Grupos do Segundo Segmento da Corte

\begin{tabular}{lcccc}
\hline & \multicolumn{2}{c}{ Grupo Caso } & \multicolumn{2}{c}{ Grupo Controle } \\
\hline \multirow{3}{*}{ Média } & Idade & APACHE II & Idade $^{1}$ & APACHE II \\
Mediana & 58 & 15 & 63 & 13 \\
Desvio-padrão & 65 & 13 & 69 & 13 \\
IC 95 & 21 & 4 & 20 & 5 \\
\hline
\end{tabular}

IC 95= intervalo de confiança de $95 \% .{ }^{1} p=0,43 .{ }^{2} p=0,71$

Tabela 2 - Distribuição dos Pacientes do Segundo Segmento Cronológico da Coorte de acordo com as Classes de Diagnóstico Primário.

\begin{tabular}{lcc}
\hline Diagnóstico Primário & $\begin{array}{c}\text { Freqüência } \\
\text { Absoluta }\end{array}$ & $\begin{array}{c}\text { Freqüência } \\
\text { Relativa }\end{array}$ \\
\hline Respiratório & 18 & $34 \%$ \\
Cardiovascular & 1 & $2 \%$ \\
Neurológico & 15 & $28 \%$ \\
Sepse não respiratória & 5 & $9 \%$ \\
Pós-operatório imediato & 5 & $9 \%$ \\
Trauma & 9 & $17 \%$ \\
Total & 53 & $100 \%$ \\
\hline
\end{tabular}




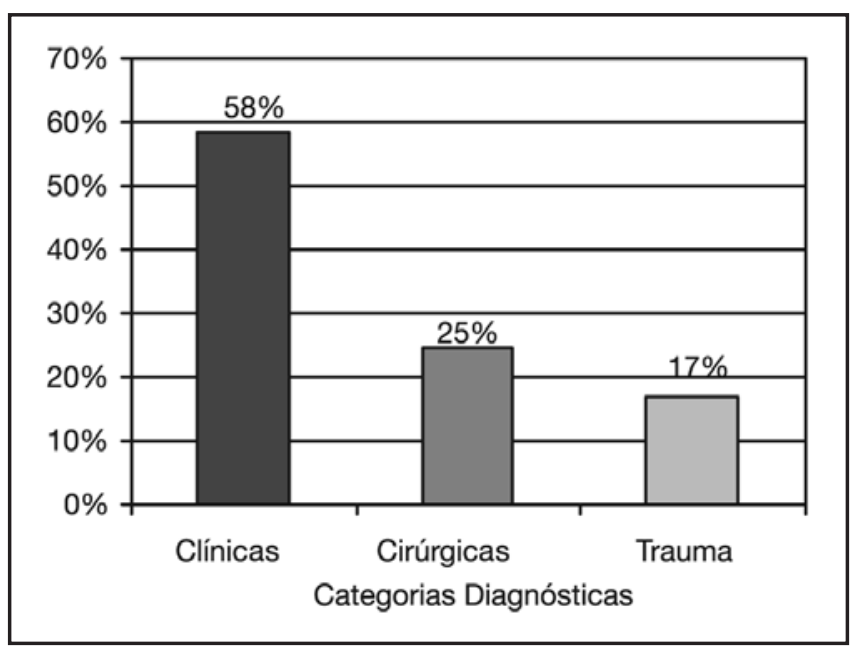

Figura 1 - Distribuição dos Pacientes no Segundo Segmento da Coorte por Categoria de Diagnósticos de Internação $(n=53)$.

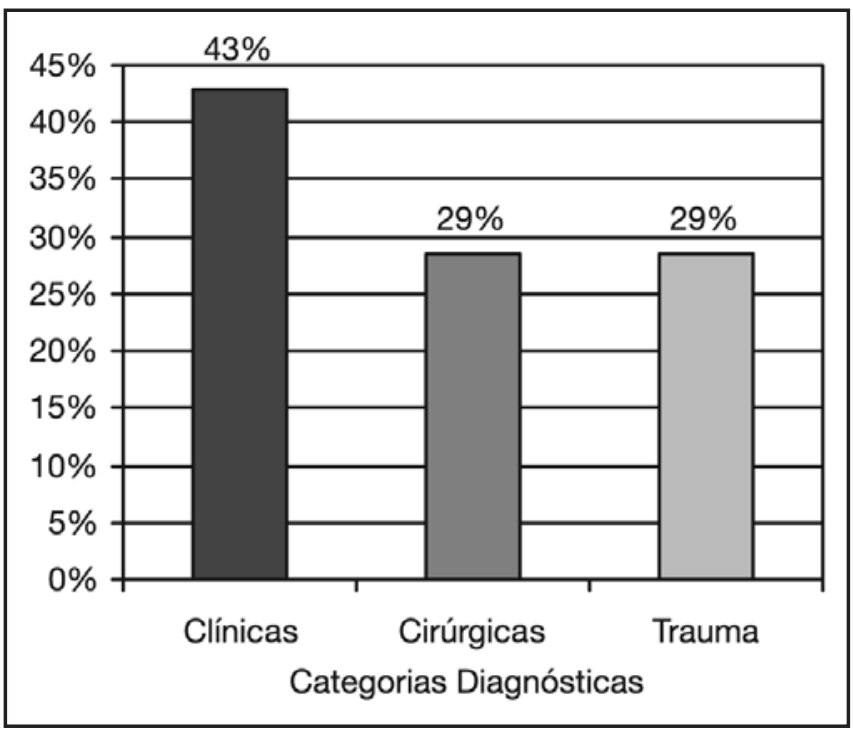

Figura 2 - População de Origem dos Pacientes com PAVM no Segundo Segmento da Coorte $(n=14)$.

As taxas de incidência nos anos de 2001 a 2003 foram respectivamente $28,05 \%$ o $12,92 \%$, $22,45 \%$ \% $10,18 \%$ e $10,75 \%$ o $\pm 7,61 \%$ o (Figura 3 ). A redução de incidência após implantação do protocolo não alcançou estatística significativa $(p=0,97)$.

A taxa de mortalidade global no segundo segmento da coorte foi $47 \%$ ( $n=53 ; I_{95}=34 \%$ a $61 \%$ ). Para os grupos controle e caso, as taxas foram respectivamente $49 \%\left(n=39 ; I_{95}=33\right.$ a $\left.65 \%\right)$ e $43 \%\left(n=14 ; I C C_{95}=\right.$ $14 \%$ a $72 \%$ ) (Figura 4). O risco relativo de morte entre os dois grupos foi $R R=0,88$ (IC ${ }_{95}=0,26$ a 2,94), sem estatística significativa $(p=0,65)$.

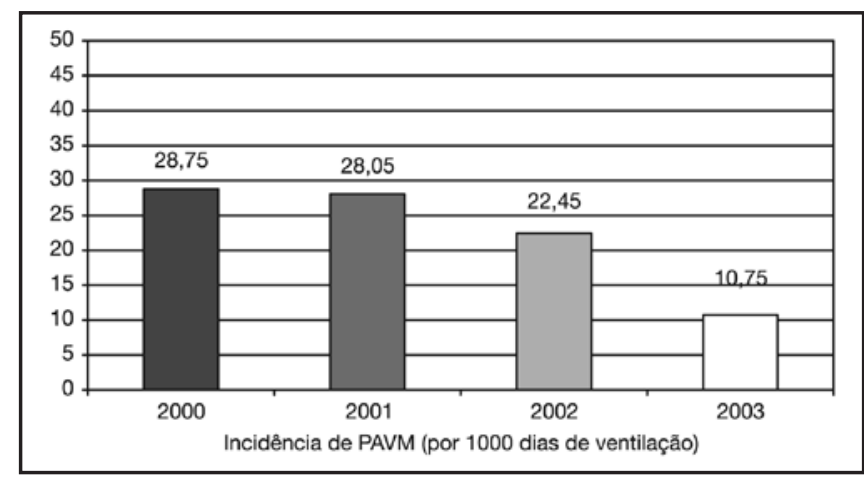

Figura 3 - Taxas de Incidência Anual do Período de 2000 a 2003 (em casos \%o).

$p=0,97$ para comparação entre as incidências de 2002 e 2003. A incidência em 2000, mostrada como referência, tem por fonte relatório da Comissão de Controle de Infecção Hospitalar do Hospital Mário Lioni.

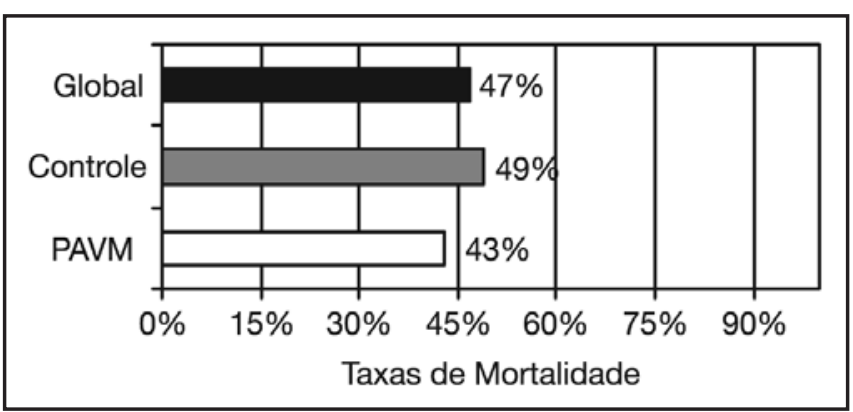

Figura 4 - Taxas de mortalidade no Segundo Segmento da Coorte.

$p=0,65$ para comparação entre o grupo caso e o controle.

Do total de casos de PAVM registrados no segundo segmento cronológico do estudo, apenas três tiveram início precoce $(21 \%)$ e oito lavados bronco-alveolares foram obtidos (57\%), 50\% destes com flora mista. Pseudomonas aeruginosa foi isolada em seis pacientes (75\%, 51\% dos lavados), Acinetobacter $s p$ em um caso (12,5\%, $8 \%$ dos lavados), MRSA em um caso (12,5\%, 8\% dos lavados), Staphylococcus aureus meticilina-sensível (MSSA) em um caso $(12,5 \%$, $8 \%$ dos lavados), outros Gram-negativos do grupo CESP (S. marcescens e E. aerogenes) em dois casos (25\%, $17 \%$ dos lavados) e Stenotrophomonas maltophilia em um caso $(12,5 \%, 8 \%$ dos lavados) (Figura $5)$. Dos oito resultados de lavados bronco-alveolares disponíveis, dois tratavam-se de PAVM precoce, um era grande queimado e outro proveniente de internação prolongada na enfermaria e antes disto em cuidados domiciliares. Este teve como agentes Pseudomonas aeruginosa e Serratia marcescens. Aquele primeiro teve por causadores Acinetobacter baumanii e Staphylococcus aureus sensível a meticilina. Entre as Pseudomonas duas mostraram perfil 


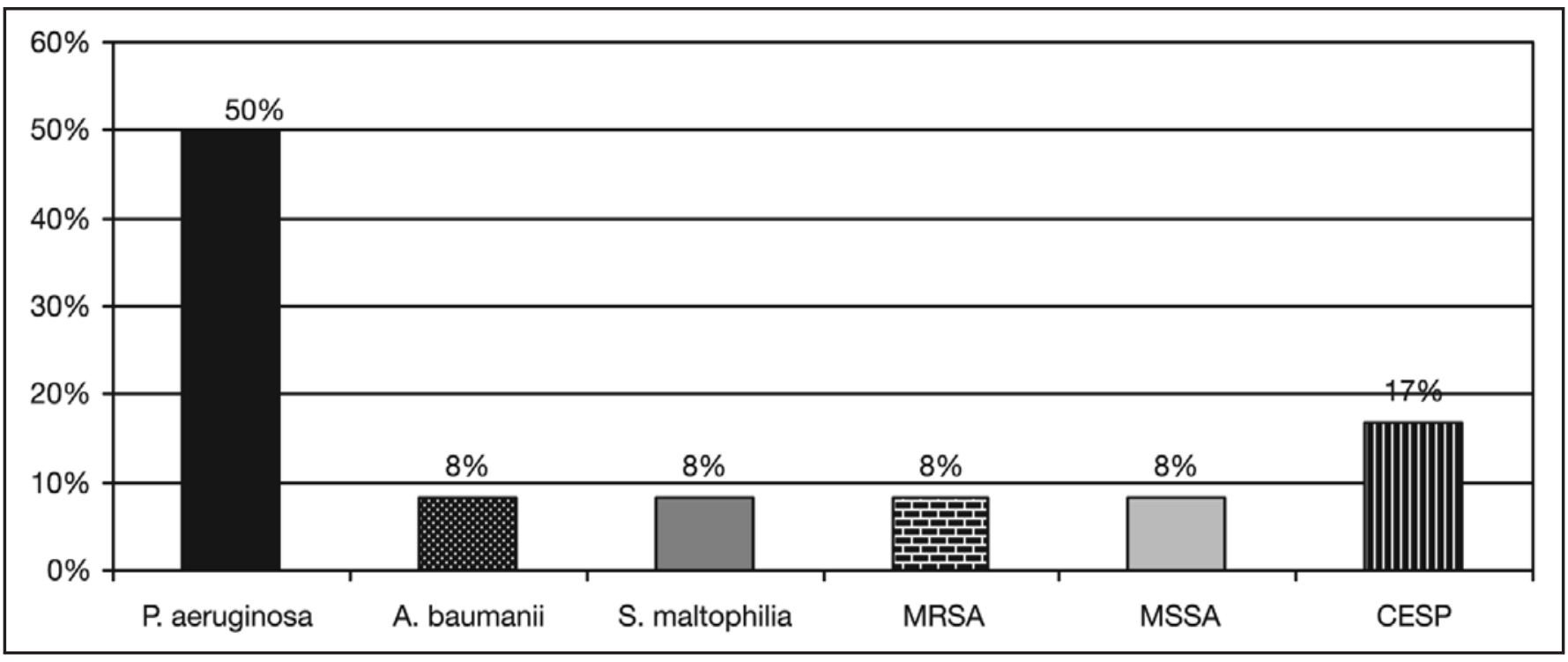

Figura 5 - Distribuição de Freqüência dos Agentes Microbiológicos Isolados durante o Segundo Segmento da Coorte. Os percentuais referem-se ao total dos resultados achados nos lavados bronco-alveolares dos pacientes com diagnóstico clínico de PAVM.

de multisensibilidade, três eram multi-resistentes e uma só sensível a polimixina B. Ambas as enterobactérias eram produtoras de betalactamase de espectro estendido.

\section{DISCUSSÃO}

Alguns estudos ${ }^{10-12}$ demonstraram consistentemente que o decúbito semi-recumbente reduz a incidência de pneumonia associada à ventilação mecânica. Estudos sugerem que a aspiração realizada com técnica estéril reduz a incidência de PAVM, tanto quanto o cuidado com a contaminação dos fluidos nos circuitos do respirador $^{2}$.

A maioria dos estudos relatados na literatura avaliando prevenção de PAVM considerou o efeito de apenas uma intervenção em reduzir a incidência dessa infecção. O que se propõe no presente estudo é uma abordagem multinível, agrupando três medidas de prevenção simples e de baixíssimo custo, até então não padronizadas nesta unidade.

Os resultados deste estudo demonstram diminuição de incidência intensa em amplitude, após a implantação do protocolo de profilaxia, em confronto com as elevadas probabilidades de erro alfa observadas. Isto pode significar de fato que a diferença de incidência obtida nesta amostra deva-se tão somente ao erro aleatório, e não diferença real na população. Em contrapartida, quanto maior o rigor para o nível de significância e me- nor o tamanho da amostra, maior a probabilidade de erro tipo beta, isto é, falso-negativo. Tendo em vista o objetivo formulado, para detecção de uma diferença de dez casos de pneumonia por mil dias de ventilação (10\%o), com erro alfa aceito em até $5 \%$ e poder estatístico de $80 \%$, necessários seriam pelo menos cento e dezesseis pacientes.

A mortalidade encontrada nos dois grupos foi semeIhante e não alcançou significância estatística. A mesma limitação quanto ao tamanho da amostra se aplica à análise desta variável de desfecho. Entretanto, tanto a pequena magnitude da diferença entre os dois grupos quanto os estreitos intervalos de confiança, indicativos de precisão nas estimativas, sugerem dever-se apenas ao erro amostral. Assim não fosse, dever-seia considerar um efeito protetor da pneumonia, caso o real escore populacional encontre-se na porção do intervalo de confiança que representa menor risco relativo de morte. Chama-se atenção para a semelhança de resultado, na avaliação desta variável de desfecho, com um estudo publicado em 2002, baseado nos dados do banco MediQuali Profile, no qual a mortalidade no grupo caso era $30,5 \%$ versus $30,4 \%$ no grupo controle com $p=0,713^{8}$.

Não foram utilizados neste estudo métodos de análise multivariável, como amostragem pareada ou regressão múltipla ${ }^{5}$, procedimentos que limitam a interferência de fatores de risco independente para mortalidade que possam funcionar como vieses, sobretudo de 
suscetibilidade ${ }^{5,15,16}$, tais como a população afetada (pacientes com diagnóstico inicial clínico versus cirúrgico), o tempo do início da pneumonia, os germes implicados, a adequação da antibioticoterapia inicial e critérios diagnósticos ${ }^{5,17}$. Foram realizados até então seis trabalhos publicados avaliando mortalidade atribuível a PAVM que fizeram uso de um grupo de controles pareados ${ }^{5-7,17-18}$. Entretanto nesta amostra não havia pares elegíveis no grupo controle para cada paciente no grupo caso, impossibilitando o emprego de tal método. Mesmo dentre os estudos que o fizeram encontra-se resultados díspares. Um deles demonstrou ausência de diferença na mortalidade ${ }^{17}$, um evidenciou pequeno incremento sem significância estatística ${ }^{18}$, dois encontraram aumento estatística e clinicamente significativo ${ }^{6,19} \mathrm{e}$ um (o mais recente e de desenho mais criterioso) mostrou apenas tendência a aumento de mortalidade ${ }^{5}$. A análise do método de cada um destes estudos revela algumas diferenças importantes que tornam difícil a comparação dos resultados.

Procedeu-se no presente estudo, todavia, ao ajustamento univariável para a gravidade de doença à admissão, aferida pelo APACHE II. No entanto, tem-se que ressaltar que pareamento feito somente com variáveis colhidas na admissão, pode não detectar diferença de gravidade entre os grupos resultante de disfunções orgânicas que se desenvolvam no decorrer do seguimento. A maioria dos casos de PAVM costuma ocorrer, em média, após o quarto dia de admissão, como foi o caso do estudo em questão. Deste modo, a menos que se pareie caso e controle usando também algum escore de disfunção orgânica tempo-dependente, a mortalidade diretamente associada a PAVM pode ser superestimada. Somente um estudo até então teve tais cuidados, de parear pelo Multiple Organ Dysfunction (MOD) escore registrado no dia anterior ao diagnóstico da infecção ${ }^{5}$, mesmo assim não demonstrou diferença significativa.

Dos estudos mencionados, dois que não encontraram risco aumentado de morte associado à pneumonia, um não arrolou pacientes clínico ${ }^{17}$ e o outro só incluiu $26 \%$ desta categoria ${ }^{18}$. Ao contrário, dos três que apontaram aumento de mortalidade, um tinha $44 \%$ de pacientes clínicos ${ }^{6}$ e o outro $63 \%{ }^{5}$. Neste último, a mortalidade absoluta observada foi 30,8\% nos pacientes clínicos versus $12,1 \%$ nos cirúrgicos ( $p=0,005)$, enquanto a mortalidade atribuível foi $65 \%$ versus $27,3 \%(p=0,04)$ em análise de sensibilidade ${ }^{5}$.

Também pode ser viés de susceptibilidade ${ }^{16} \mathrm{O}$ im- pacto de germes multi-resistentes. ${ }^{6}$ Tomadas as três coortes que avaliaram mortalidade de maior tamanho e melhor desenho $0^{5-7}$, as duas mais recentes continham uma proporção de organismos multi-resistentes de $23 \%^{7}$ e $24 \%^{5}$ respectivamente. Fagon e col. encontraram mortalidade atribuível de $42,8 \%$ $\left(\mathrm{IC}{ }_{95}=14,5 \%\right.$ a $\left.69 \%\right) \mathrm{com} \mathrm{RR}=2,5\left(\mathrm{IC}{ }_{95}=1,31 \mathrm{a}\right.$ $4,61)$ nos pacientes que desenvolveram PAVM por $P$. aeruginosa ou Acinetobacter sp em relação ao total de pacientes ${ }^{6}$. Já Cook e col. mostraram mortalidade atribuível semelhante nos grupos com PAVM devido a germes de alto $(33,3 \%)$ e baixo risco $(32 \%$; $p>$ $0,05)^{5}$. Na coorte que avaliou somente a população de PAVM tardia, os autores acharam mortalidade de $65 \%$ no subgrupo com PAVM tardia devido a patógenos multi-resistentes comparada com $31,3 \%$ no subgrupo causado por outros germes $(R R=2,07$; IC ${ }_{95}=1,29$ a 3,$\left.35 ; p<0,05\right)$ ou mesmo $37,4 \%$ no subgrupo sem PAVM tardia $\left(R R=1,74 ; I_{95}=1,21 \mathrm{a}\right.$ $2,50 ; p<0,05)$. Flora resistente persistiu como fator de risco independente na análise multivariável realizada no total de pacientes $\left(R R=3,4 ; I_{95}=1,2\right.$ a 10 ; $\mathrm{p}=0,025)$ e no grupo com PAVM tardia ( $\mathrm{RR}=5,4$; IC ${ }_{95}=2,8$ a 10,$\left.3 ; p=0,009\right)^{7}$.

A realização do lavado bronco-alveolar não parece alterar o prognóstico ${ }^{5,14}$ quando se corrige a antibioticoterapia começada empiricamente ${ }^{14}$. Esta sim, quando apropriada desde o início é que tem impacto favorável nos desfechos clínicos ${ }^{14,19}$. Nesta amostra, no ano de 2003, a maioria dos germes isolados nos lavados é de alto risco para resistência a antimicrobianos, mesmo os dois casos de PAVM precoce culturados, os quais, um era grande queimado e outro proveniente de tratamento prolongado em regime domiciliar. Salienta-se assim a importância de avaliar outros demográficos de cada paciente-caso além da precocidade do início da infecção, bem como a importância da vigilância do perfil microbiológico da unidade, para confecção de adequada estratégia inicial de antibioticoterapia, com vistas à redução de mortalidade e de emergência de cepas resistentes.

Dentre os casos de pneumonia com confirmação microbiológica nesta coorte, o esquema antimicrobiano prescrito a priori foi adequado em todos os casos menos um, aquele ocasionado por Pseudomonas aeruginosa só sensível a polimixina $B$.

\section{CONCLUSÃO}

Os resultados deste estudo sugerem que a aquisição 
de PAVM não se associa à diferença de mortalidade estatisticamente significativa.

A incidência de PAVM mostrou importante tendência ao declínio após a implantação do protocolo de profilaxia, porém sem significado estatístico.

A flora microbiana isolada tem distribuição probabilística que não difere relevantemente da comumente apresentada em séries de caso relatadas na literatura. $O$ registro do perfil de sensibilidade da microbiota local e sua divulgação, através de relatórios internos periódicos, permitem a otimização da terapia antimicrobiana empírica inicial, com vistas a reduzir a mortalidade e a emergência de cepas multi-resistentes.

É necessário re-analisar, prospectivamente, maior amostra, para atingir poder estatístico suficiente para conclusões definitivas acerca do prognóstico da PAVM, incluindo medidas de controle e identificação de covariáveis de risco de morte que funcionem como vieses.

\section{ABREVIATURAS}

APACHE II - Acute Physiology and Chronic Health EvaIuation II

CDC - Center for Disease Control and Prevention

DP - desvio-padrão

EPIC - European Prevalence of Infection in Intensive Care Study

Grupo CESP - Citrobacter, Enterobacter, Serratia e Proteus.

$\mathrm{IC}_{95}$ - intervalo de confiança de $95 \%$

MOD - Multiple Organ Dysfunction

MRSA - Staphylococcus aureus meticilina-resistentes MSSA - Staphylococcus aureus meticilina-sensivel NNIS - National Nosocomial Infection Survaillance System PAVM - pneumonia associada à ventilação mecânica $\mathrm{RR}$ - risco relativo

\section{REFERÊNCIAS}

01. National Nosocomial Infections Surveillance (NNIS) System report: data summary from January 1990-May 1999. Am J Infect Control, 1999;27:520-532.

02. Fernandes AT, Zamorano PO, Torezan Filho MA - Pneumonia Hospitalar em: Fernandes AT - Infecção Hospitalar e suas Interfaces na Área da Saúde. São Paulo, Atheneu, 2001;21:523

03. Vincent JL, Bihari DJ, Suter PM et al - The prevalence of nosocomial infection in intensive care units in Europe. Results of the European Prevalence of Infection in Intensive Care (EPIC) Study. JAMA, 1995;274:639-644.

04. Prade SS - Estudo brasileiro da magnitude das infecções hospitalares em hospitais terciários. Rev Controle Infec Hosp, 1995;2:11-24.

05. Heyland DK, Cook DJ, Griffith L et al - The attributable morbidity and mortality of ventilator-associated pneumonia in the critically ill patient. The Canadian Critical Trials Group. Am J Respir Crit Care Med, 1999;159:1249-1256.

06. Fagon JY, Chastre J, Hance AJ et al - Nosocomial pneumonia in ventilated patients: a cohort study evaluating attributable mortality and hospital stay. Am J Med, 1993;94:281-288.

07. Kollef MH, Silver P, Murphy DM et al. The effect of late-onset ventilator-associated pneumonia in determining patient mortality. Chest, 1995;108:1655-1662.

08. Rello J, Ollendorf DA, Oster G et al - Epidemiology and outcomes of ventilator-associated pneumonia in a large US database. Chest, 2002;122, 2115-2121.

09. Roderjan CN, Souza PCP, Lugarinho ME et al. Na contramão da medicina baseada em evidências: posição semi-recostada em pacientes sob ventilação mecânica versus pneumonia. RBTI, 2004;16:34-39.

10. Drakulovic MB, Torres A, Bauer TT et al - Supine body position as a risk factor for nosocomial pneumonia in mechanically ventilated patients: a randomised trial. Lancet, 1999;354:1851-1858.

11. Orozco-Levi M, Torres A, Ferrer M et al - Semirecumbent position protects from pulmonary aspiration but not completely from gastroesophageal reflux in mechanically ventilated patients. Am J Respir Crit Care Med, 1995;152:1387-1390.

12. Torres A, Serra-Batlles J, Ros E et al - Pulmonary aspiration of gastric contents in patients receiving mechanical ventilation: the effect of body position. Ann Intern Med, 1992;116:540-543.

13. Garner JS, Jarvis WR, Emori TG et al - CDC definitions for nosocomial infections, 1988. Am J Infect Control, 1988;16:128-140.

14. Luna CM, Vujacich P, Niederman MS et al - Impact of BAL data on the therapy and outcome of ventilator-associated pneumonia. Chest, 1997; 111:676-685.

15. Fletcher R, Fletcher SW, Wagner EH - Acaso, em: Fletcher R, Fletcher SW, Wagner EH - Epidemiologia Clínica. $3^{a}$ Ed, Porto Alegre, Artes Médicas, 1996;9:141:211-213.

16. Fletcher R, Fletcher SW, Wagner EH - Prognóstico, em: Fletcher R, Fletcher SW, Wagner EH - Epidemiologia Clínica. $3^{a}$ Ed, Porto Alegre, Artes Médicas, 1996;6:132-133.

17. Baker AM, Meredith JW, Haponik EF - Pneumonia in intubated trauma patients. Microbiology and outcomes. Am J Respir Crit Care Med, 1996;153, 343-349.

18. Papazian L, Bregeon F, Thirion X et al - Effect of ventilator-associated pneumonia on mortality and morbidity. Am J Respir Crit Care Med, 1996;154:91-97.

19. Kollef $\mathrm{MH}$, Sherman $\mathrm{G}$, Ward $\mathrm{S}$ et al - Inadequate antimicrobial treatment of infections: a risk factor for hospital mortality among critically ill patients. Chest, 1999;115:462-474. 